\title{
Dissolution Highlights from the 2016 AAPS Annual Meeting in Denver
}

\author{
Nikoletta Fotaki ${ }^{1,}{ }^{*}$, Deblina Biswas ${ }^{2}$, Susan D’Souza ${ }^{3}$, Johannes Krämer ${ }^{4}$, Xujin Lu ${ }^{5}$, Limin Zhang ${ }^{5}$ \\ ${ }^{1}$ Department of Pharmacy and Pharmacology, University of Bath, UK \\ ${ }^{2}$ Virginia Commonwealth University, USA \\ ${ }^{3}$ Tesaro Inc., USA \\ ${ }^{4}$ PHAST, Germany \\ ${ }^{5}$ Drug Product Science and Technology, Bristol-Myers Squibb Company, USA \\ e-mail:n.fotaki@bath.ac.uk
}

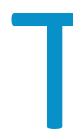
he American Association of Pharmaceutical Scientists (AAPS) held its Annual Meeting and Exposition at the Convention Center in Denver, Colorado, on November 13-17, 2016. Pre-conference workshops took place November 12-13, 2016. The meeting is a premier gathering of pharmaceutical scientists from around the world and works to address the needs of the attendees, including members of over 40 Focus Groups in nine Sections, or scientific disciplines, including two focused on dissolution testing (the In Vitro Release and Dissolution Testing and QbD and Product Performance groups).

For those with an interest in dissolution testing, there was a pre-conference workshop on "Locally acting drug products: Bioequivalence challenges and opportunities", symposia on "In silico modeling and predictive dissolution for accelerated oral drug development", "Performance test and analytical challenge of peptide nanoparticles for parenterals", "Establishing release testing for nonoral controlled release technologies", and a dialog and debate session on "Strategic dissolution approaches to enable continuous manufacturing". There were face-toface meetings of the two focus groups mentioned and the Dissolution Discussion Group. Details about some of these events are presented in this review.

\section{WORKSHOP ON “LOCALLY ACTING DRUG PRODUCTS: BIOEQUIVALENCE CHALLENGES AND OPPORTUNITIES"}

This two-day, pre-conference workshop was organized and moderated by Isadore Kanfer (Rhodes University, South Africa) and Barbara Davit (Merck, USA). During this workshop, mostly all locally acting drug products and their in vivo performance testing were covered, and the perspective of meaningful drug release/dissolution testing was discussed. More information related to the state of the biopharmaceutical and regulatory science of drug products for dermal applications were reported as the outcome of a USP workshop on "Quality attributes of drug products applied to the skin" (September 21$22,2015)$. Unlike for systemically acting drug products, the bioavailability of locally acting drug products does not always serve as the surrogate parameter for their therapeutic efficacy. From the perspective of in vitro performance testing, one of the most relevant presentations was given by Keith Gallicano (Novum Pharmaceutical Research Services, USA). He concluded that there is direct evidence from topical nonsteroidal anti-inflammatory drug (NSAID) pharmacokinetic studies that the drug arrives at the site of action (synovial fluid) mainly via the distribution of the drug through the systemic system rather than by direct penetration from the application site, and that plasma and synovial fluid NSAID concentrations are correlated following topical administration. Therefore, in vivo drug release testing is not always linked to the in vivo performance of the drug products. Nevertheless, in vitro release testing provides meaningful information on the quality of the drug product.

Sid Bhoopathy (Absorption Systems, USA) described the principles of in vitro equivalence as a surrogate for equivalence in the rate and extent of drug absorption. Release rates are determined by the composition and the quality of the drug product, which are impacted by the following variables:

1. Physical and chemical properties of the formulation

2. Formulation preparation steps

3. Manufacturing process

4. Excipient quality

In the case of semisolids, drug release testing follows meaningful characterization by quality attributes such as globule size distribution, viscosity profile, $\mathrm{pH}$, zeta potential, osmolality, surface tension, and drug distribution in different phases for emulsion-type semisolids. For the suspension-type semisolids, the typical attributes are crystalline habit, appearance, $\mathrm{pH}$, specific gravity, osmolality, surface tension, buffer 
capacity, viscosity, redispersibility, soluble fraction of the active pharmaceutical ingredient (API) in the final drug product, unit dose content, and particle size distribution. For in vitro release and absorption testing the IDAS (in-vitro dissolution absorption system AAPS poster \# 22R1030) was introduced which combines process and effect. Dennis Bashaw (Food and Drug Administration [FDA], USA) presented examples of locally acting drugs for the treatment of gastrointestinal (GI) diseases. Multipoint dissolution profiles (i.e., multimedia dissolution testing) were compared with targeted in vivo area under the curve (AUC) trunks. He stated that "for drugs delivered as modified release formulations, it is important to consider solubility in different regions of the gut as significant differences can arise which will ultimately influence drug bioavailability. Stoma fluids provide useful information on drug solubility and are more reflective of the in vivo conditions than compendia buffers that are typically used. However, due to their variability and the multitude of factors they are influenced by, they are not fully representative of GI luminal fluids." For drugs applied to the lung, Günther Hochhaus (University of Florida, USA) presented a dissolution apparatus built based on physiology findings consisting of a modified Transwell system approach and dissolution medium containing 0.5 $\%$ Tween. For selected drugs, a correlation between the mean dissolution time and the mean absorption time (MAT) were established. He also introduced an algorithm to predict the in vivo dissolution rate based on the outcome of cascade impactor studies. His presentation concluded with a comparison of the European Medicines Agency and FDA regulatory decision pathways.

\section{SYMPOSIUM ON "IN SILICO MODELING AND PREDICTIVE DISSOLUTION FOR ACCELERATED ORAL DRUG DEVELOPMENT"}

A symposium on "In silico modeling and predictive dissolution for accelerated oral drug development" was held on Monday afternoon organized and moderated by Xujin Lu (BMS, USA) and Nikoletta Fotaki (University of Bath, UK). Raimar Loebenberg (University of Alberta, CA) gave a presentation on "In Vitro and In Vivo Correlations and Biorelevant Dissolution Using Computers Simulations in a QbD Environment." Initially, he discussed the fundamentals of Quality by Design (QbD) and the Biopharmaceutics Classification System (BCS), noting that $\mathrm{BCS}$ can be seen as the scientific, mechanistic approach which enables the industry to apply QbD to their product development. Understanding critical product attributes in vitro and in vivo is needed for a successful QbD strategy, and tablet disintegration and dissolution are fundamental dosage form properties that can be used as performance tests. He emphasized that the dissolution process can be governed by either the API properties or the interaction of excipients and the API, and, depending on the drug release mechanism, dissolution or disintegration should be chosen as a performance test. He continued his presentation with a discussion on how clinically relevant product specifications can be identified, and he presented an example with lysosomal trapping and permeability vs dissolution controlled absorption. The second presentation, entitled "Biorelevant Dissolution Measurements and Modeling at the Drug Solubility Limit: Optimizing Exposure Rankings," was given by Paul Harmon (Merck, USA). He started his presentation with a discussion on the challenges for optimizing the bioavailability in terms of the prediction of relative bioavailability of different formulations of the same BCS II/IV drug and the understanding of the formulation composition and processes that impact the biorelevant dissolution rate, and hence, AUC. He discussed the use of MiMBA (Microscopic Mass Balance Approach) on the role of API particle size in optimizing AUC. He emphasized that quality control (QC) dissolution and typical biorelevant dissolution using the whole dosage form in volumes of $500-900 \mathrm{~mL}$. He proposed the use of dispersed API particle dissolution models which can be very sensitive to particle size if the drug is kept at or below the solubility limit in biorelevant media (1X). He concluded his talk by presenting formulation examples of the biorelevant " $1 X$ " methodology and clinical outcomes. The last talk in this symposium was delivered by Kimberly Raines-Isler (FDA, USA) on "Biorelevant Dissolution and Drug Absorption Using Predictive Modeling Tools: Regulatory Perspective and Experience." At the start of her presentation, she emphasized the emerging use of biorelevant dissolution and physiologically based pharmacokinetic (PBPK) modeling as a biopharmaceutics tool to predict the impact of the factors that can influence oral drug absorption and facilitate drug product development. She described what biorelevant dissolution testing is, the applications of PBPK modeling and how the dissolution model links to a mechanistic absorption model. She pointed out the challenge of in vivo link to quality and the opportunities to leverage the use of biorelevant media allowing for better simulated conditions of the GI tract and the use of in silico absorption modeling to assess the impact of in vitro dissolution on in vivo performance. She presented two regulatory examples on the use of PBPK modeling on ensuring suitable clinical performance of batches and setting clinically relevant drug product in vitro dissolution acceptance criteria. She concluded her presentation 
with the considerations for PBPK model submission supporting a Clinically Relevant Dissolution and with future directions.

\section{SYMPOSIUM ON "PERFORMANCE TEST AND ANALYTICAL CHALLENGE OF PEPTIDE IN NANOPARTICLES FOR PARENTERALS"}

A symposium entitled "Performance test and analytical challenge of peptide nanoparticles for parenterals" was organized and moderated by Xujin Lu (Bristol-Myers Squibb, USA), Eliza Fung (Bristol-Myers Squibb, USA) and Susan D'Souza (Tesaro, Inc., USA). The session reviewed the challenges with the development of performance tests for nanoparticles and provided a forum for scientists from industry, regulatory agencies, and academia to discuss issues encountered in practice, and potential approaches to address these. Beata Sweryda-Krawiec (Tarveda Therapeutics, USA), the first speaker, described some common challenges observed with measurement of in vitro release from nanoparticles, especially the identification of the free drug from the encapsulated drug. She presented case studies that walked the audience through the development and evaluation of a solid-phase microextraction technique (activated carbon extraction [ACE]) where the determination of free/total drug ratio, physical stability of the formulation at different conditions, process evaluation, and in vivo effectiveness were demonstrated. Johannes Kramer (PHAST, Germany), the second speaker, presented the talk on behalf of Alexis Guillot (PHAST, Germany). In his talk, Dr. Kramer discussed the development and validation of a solid phase extraction technique to measure free and entrapped drug concentration from lipid nanoparticles (Lipidot ${ }^{\circledR}$ ). His talk highlighted major challenges with evaluating drug release from nanoparticles and concluded with the demonstration of a fiber optic method to assess in vitro release from a variety of nanoparticle drug products. Banu Zolnik (FDA, USA) began her talk by outlining the importance of in vitro dissolution testing of nanoparticles as a tool during formulation development, for QC, biowaiver purposes, and post-approval manufacturing changes. In her talk, she highlighted the differences in the acceptance criteria for immediate and extended release nanoparticulate products. She stated that the common deficiencies identified by the agency in submissions are that the in vitro release method development is not included in the submission, method validation issues, analytical method issues (measuring encapsulated vs released drug), discriminatory ability of the method is not demonstrated, and data do not support the proposed acceptance criterion. Following the presentations, the discussion was led by the moderators. The Q\&A session that followed was engaging; the audience requested additional information on the techniques presented, as well as the speakers' thoughts on some of the challenges faced during in vitro release method development and validation for nanoparticles.

\section{SYMPOSIUM ON "ESTABLISHING RELEASE TESTING FOR NON-ORAL CONTROLLED RELEASE TECHNOLOGIES"}

This symposium was organized and moderated by Benjamin Maynor (Liquidia Technologies, USA) and Deblina Biswas (Virginia Commonwealth University, USA). The session focused on the development of functional release testing methodologies for long-term dosage forms. Such long-term delivery agents often require dissolution/release tests that are discriminatory but at the same time benefit from short-term QCbased methods. Short-term QC-based tests would be both time and cost feasible, but they are also required to be predictive and with a good correlation with their normal release rate profiles. Such QC development methodologies focusing on release testing form the crux of a critical quality attribute of the drug product. The symposium was started with a presentation by Matthew S. Halquist (Virginia Commonwealth University, USA) on "Relationship between Functional Drug Release Testing and QC-Based Discriminating Test Methods." He discussed the various standardized and conventional methods used for release testing of non-oral controlled-release dosage forms: USP-IV, dialysis methods, sample and separate, and their attributes and relevance. Various QC requirements such as media and sink conditions, sampling frequency, robustness, and sensitivity requirements of the analytical methods involved in developing such a test were emphasized. He also stressed the need for a release method to be not only predictable but also practical to use as well as discriminatory to distinguish between formulation changes and detect inter-batch variability in the manufacturing process. Finally, he offered his closing thoughts on biorelevant considerations for development of noncompendial in vitro devices/methods in special cases such as implants using two case studies and discussed the performance of these models in predicting release. The second presentation in the symposium was on the topic of "Mathematical Modeling of Diffusion in Controlled Release Dosage Forms" and was given by Juergen Siepmann (University of Lille, France). His talk emphasized mathematical modeling as an effective tool to determine diffusional mass transport of non-oral controlled-release dosage forms. Release of 
small and large molecules such as peptides from PLGA microparticles and lipid implants were discussed as case studies. Emphasis was placed on the variability in drug release mechanisms and kinetics in the above cases and how crucial parameters like microparticle size and polymer degradation impacted the drug diffusion mechanism from the polymer scaffold. Simple quantitative mathematical theories were developed sequentially into complex mathematical formulas taking into consideration the above crucial parameters. These theories were then applied for correlation with experimental data to show the applicability of in silico simulations in being both time and cost effective, especially in the case of extended controlled release dosage forms. He concluded the presentation by suggesting the preference of simpler models, the evaluatione of model fittings with caution, and prevention of over-parameterization in a model. The concluding presentation of the symposium was given by Jeff Kindig (Liquidia Technologies, USA) on "Dissolution Testing for Depot/Implant Drug Delivery Systems." His talk introduced the currently available USP guidelines for injections and implanted drug products. He discussed the various spectrum of factors to consider while developing a QC drug release test such as solubility of the drug in the media, sink conditions, biorelevance of the media, and stability of the drug in the release media. He also focused on accelerated release testing with maintenance of discriminatory ability of the method. Finally, case studies of engineered nanoparticles and microparticles were discussed using an innovative PRINT ${ }^{\circledR}$ technology, followed by discussion about the development of a QC batch release discriminating method for long-acting depots with a focus on multiple formulations, API stability, and analytical method sensitivity.

\section{DIALOG AND DEBATE SESSION ON "STRATEGIC DISSOLUTION APPROACHES TO ENABLE CONTINUOUS MANUFACTURING"}

This dialog and debate session was organized by Limin Zhang (Bristol-Myers Squibb Company, USA) and comoderated by Nikolay Zaborenko (Eli Lilly \& Co.). It was designed to stimulate discussions on building dissolution knowledge on drug products through strategic approaches that could lead to real-time release testing which enables the reduction or the elimination of end-product testing and provides increased assurance of drug product quality. Continuous manufacturing (CM) has been proposed as a path forward by the FDA in the context of $\mathrm{QbD}$. The implementation of process analytical technology (PAT) for process control and utilizing process knowledge such as input material attributes, particle size distribution from granulation, and weight and hardness from compression to build the correlations between material/process attributes and in vitro dissolution release through modeling approach are encouraged by regulatory agency and studied by academia and industry. This session promoted extensive discussion among industry experts and the regulatory agency on the strategies and approaches of developing dissolution models in continuous manufacturing. Dr. Min Li (FDA/CDER/OPQ/ONDP, USA) gave a presentation entitled "Dissolution for Real Time Release Testing (RTRT) in Continuous Manufacturing (CM)" that focused on the strategies and approaches on dissolution model development. Such strategies include but are not limited to product and process understanding, identifying critical material attributes (CMA), and process parameters (CPP) in CM that could impact the in vitro dissolution performance, implementing PAT and constructing dissolution models that use multivariate analysis (e.g., Principle Component Analysis/Principle Component, and Regression, Partial Least Square) to correlate process and/or material data with dissolution. She outlined the approaches for dissolution model development, model validation, and model maintenance and provided two case studies to demonstrate the approaches. In the first case study, Principle component analysis (PCA) was used to decompose the variants in pre-treated NIR data that were generated from a continuous tablet manufacturing via direct compaction with four variables at three levels. Dissolution data were generated using reference method and fitted by both model independent and model dependent approaches. Multilinear regression was performed between NIR data and dissolution data, and the selected model was validated using multiple independent datasets. The second case study demonstrated the use of modified Noyes-Whitney Equation where dissolution rate (Z) was predicted by granule particle size distribution, drug content, and water content in the final blend, tablet weight, thickness, and hardness using a partial least square model with four factors. The model was calibrated with the experimental data obtained from the batches encompassing the typical manufacturing space for all model input variables, and external validation was performed with independent batches produced at processing conditions that span the design space for important parameters. She concluded the presentation with the desire for linking the dissolution model with a mechanistic absorption model for in vivo performance prediction. Yleana Colon, on behalf of Sarah Nielsen (Janssen, USA), started her presentation by emphasizing the importance of switching from batch 
process to continuous manufacturing and the advantages of using real-time release testing approach through inprocess physical testing and NIR monitoring at each critical process step over traditional release approach via design of experiment (DOE) and quality attributes end-point testing. She shared a representative scheme of the continuous drug product manufacturing line and the real-time release testing strategy for the critical process steps. To expand the real-time release testing to dissolution, she described considerations on generating desired dissolution data using risk assessment and DOE approaches and building dissolution models using decision trees. She also described reference dissolution method development and validation, dissolution model maintenance when raw material supplier changes, ongoing verification of models and sampling strategy for continuous manufacturing. She concluded her presentation by citing various dissolution modeling approaches from the literature. During the $Q \& A$ session, a question was raised about the minimum requirement to have a predictive dissolution model. FDA speakers sitting in the debate session shared their personal opinions that it requires the applicant to demonstrate the evaluation results of the impact of CMAs and CPPs on dissolution profiles. The applicant could leverage a risk-based approach to choose which parameters to test and which parameters not to test based on BCS and risk assessment.

\section{IVRDT FOCUS GROUP}

\section{FACE-TO-FACE MEETING}

The annual face-to-face meeting of the focus group was held on Monday, November 14. The current chair, Nikoletta Fotaki (University of Bath, UK), reviewed the accomplishments of the focus group and described the objectives set for 2017. Xujin Lu (BMS, USA) presented the AAPS E-course organized and delivered by members of the focus group and presented the summary of the outreach workshop in China (June 2016). The student outreach activities were presented by Deblina Biswas (Virginia Commonwealth University, USA). Vivian Gray (Dissolution Technologies, USA) gave updates on USP aspects and Dissolution Technologies activities and upcoming articles. Susan D'Souza (Tesaro Inc., USA) presented the sessions of interest at the annual meeting. A lively discussion by all attendees on proposing ideas and points to consider for future activities of the focus group followed. 